\title{
Correction to: SGLT2 Inhibitors in Older Adults with Heart Failure with Preserved Ejection Fraction
}

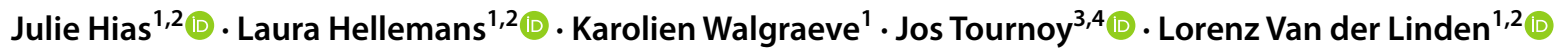

Published online: 16 February 2022

(c) The Author(s), under exclusive licence to Springer Nature Switzerland AG 2022

\section{Correction to: Drugs \& Aging}

https://doi.org/10.1007/s40266-022-00920-7

In the original online version of the article Section 3.3 Clinical Relevance of the EMPEROR-Preserved Trial for Older Adults with HFpEF, the third sentence of the first paragraph, which currently reads

First, in EMPEROR-Preserved, the time to a first hospital admission for heart failure was reduced, but in absolute terms not to the same extent as was the case in EMPEROR-Reduced.
Should read:

First, in EMPEROR-Preserved, the time to a first hospital admission for heart failure was prolonged, but in absolute terms not to the same extent as was the case in EMPEROR-Reduced.

The original article has been corrected.

The original article can be found online at https://doi.org/10.1007/ s40266-022-00920-7.

Lorenz Van der Linden

lorenz.vanderlinden@uzleuven.be

1 Pharmacy Department, University Hospitals Leuven, Leuven, Belgium

2 Department of Pharmaceutical and Pharmacological Sciences, KU Leuven, Leuven, Belgium

3 Department of Geriatric Medicine, University Hospitals Leuven, Leuven, Belgium

4 Geriatrics and Gerontology, Department of Public Health and Primary Care, KU Leuven, Leuven, Belgium 